\title{
Effect of $\beta$-globin MAR characteristic elements on transgene expression
}

\author{
${\text { QIN } \text { LI }^{1 *}, \text { WEIHUA DONG }}^{2 *}$, TIANYUN WANG ${ }^{2 *}$, ZHONGHE LIU ${ }^{3}$, FANG WANG $^{2}$, \\ XIAOYIN WANG ${ }^{2}$, CHUNPENG ZHAO $^{2}$, JUNHE ZHANG ${ }^{2}$ and LI WANG $^{2}$ \\ ${ }^{1}$ Laboratory of Analytical and Testing, and ${ }^{2}$ Department of Biochemistry and Molecular Biology, \\ Xinxiang Medical University, Henan 453003; ${ }^{3}$ Department of Orthopedics, First Affiliated \\ Hospital of Xinxiang Medical University, Weihui, Henan 453100, P.R. China
}

Received November 25, 2012; Accepted April 2, 2013

DOI: $10.3892 / \mathrm{mmr} .2013 .1424$

\begin{abstract}
The aim of the present study was to investigate the effect of the characteristic elements of matrix attachment region (MAR) on transgene expression. Human $\beta$-globin MAR was obtained by PCR amplification. A splicing MAR fragment containing all the characteristic elements of $\beta$-globin MAR was artificially synthesized and then cloned into the eukaryotic expression vector. Following digestion and sequence identification, we transfected Chinese hamster ovary (CHO) cells with the two vectors, and then screened for the transformation of stable cells. The transgene expression level was analyzed by ELISA, and the copy numbers of the CAT gene were analyzed by real-time fluorescent quantitative PCR. $\beta$-globin MAR enhanced CAT reporter gene expression by 2.1489-fold, whereas the $\beta$-globin MAR characteristic elements did not enhance this expression. The real-time fluorescent quantitative PCR analysis demonstrated that the relative copy numbers of the CAT gene of the $\beta$-globin MAR expression vector were 1.2-fold higher compared with those of the non-MAR expression vector. MAR was able to improve the transgene expression level to a certain extent. The MAR characteristic elements did not improve the transgene expression alone. The transgenic expression levels were not linear with the transgene copy number; however, the enhancement of transgenic expression was relative to the increase in the gene copy number.
\end{abstract}

\section{Introduction}

With the development of modern biotechnology, transgene technology has been widely used in genetic engineering and

Correspondence to: Dr Tian-Yun Wang, Department of Biochemistry and Molecular Biology, Xinxiang Medical University, 601 Jinsui Road, Xinxiang, Henan 453003, P.R. China

E-mail:wtianyuncn@126.com

"Joint senior authorship

Key words: matrix attachment region, characteristic elements, transgene expression, $\mathrm{CHO}$ cells genetic therapy fields. However, low or silenced transgene expression has become a common phenomenon $(1,2)$ that limits the use of transgene technology. Certain DNA elements have been used to overcome transgene silencing and to increase transgene expression $(3,4)$, such as matrix attachment region (MAR) (5-7).

MAR is a unique DNA sequence capable of attaching to the nuclear matrix in eukaryotic chromatin. MAR is 300-1,000 bp long, and contains the topoisomerase consensus sequence and other AT-rich sequence motifs (8). Previous studies demonstrated that MAR is able to overcome transgene silencing, increase transgene expression and decrease the expression difference in different transformants, as a boundary regulatory element (9-11). In previous studies we demonstrated that human $\beta$-globin MAR is capable of efficiently enhancing transgene expression (12), and the transgene expression level may be highly increased when two $\beta$-globins flank the CAT expression cassette (13). Moreover, different MARs that flank the transgene are able to significantly increase the transgene expression level (14). As a cis-acting DNA element, MAR is capable of enhancing transgene expression, as it contains several characteristic motifs, such as the AATATATTT motif, the topoisomerase-II-binding site and an Alu element. However, the function of these motifs in enhancing gene expression, whether these motifs are able to enhance gene expression and identification of the MAR elements that are capable of increasing gene expression remain to be elucidated. In this study, we combined the characteristic elements of $\beta$-globin MAR, the AATATATTT motif, the topoisomerase-II-binding site and an Alu element, and removed the intermediate DNA connection for the synthesis of an artificial splicing of the MAR fragment to investigate the function and mechanism of the MAR characteristic elements in regulating transgene expression. Moreover, we examined the effect of the characteristic elements of $\beta$-globin MAR on transgene expression.

\section{Materials and methods}

MAR cloning and artificial synthesis. The fragment of human $\beta$-globin MAR (Genebank No. L22754) was amplified by PCR using the primers: P1: 5'-ATCGGTACC 
GTAAGACATCACCTTGCATTT-3' and P2: 5'-GCAACGCG TCATAGTTTGAGTTACCCTTT-3'. Human $\beta$-globin MAR, $\sim 2.15 \mathrm{~kb}$ in length (site 840-2,998), was amplified using human peripheral blood as a template. The $\mathrm{KpnI} / \mathrm{BglII}$ enzyme sites (GTCAGACTC and AGCGGTACC) with three additional nucleotides were inserted at the 5 ' site of the above primers, respectively. The PCR conditions were: $95^{\circ} \mathrm{C}$ for $3 \mathrm{~min}, 94^{\circ} \mathrm{C}$ for $40 \mathrm{sec}, 56-60^{\circ} \mathrm{C}$ for $30 \mathrm{sec}, 72^{\circ} \mathrm{C}$ for $2 \mathrm{~min}$ at two cycles every annealing temperature, 20 cycles at $55^{\circ} \mathrm{C}$ and a final extension at $72^{\circ} \mathrm{C}$ for $5 \mathrm{~min}$.

The characteristic elements of the human $\beta$-globin MAR, including the AATATATTT motif (sites 840-848, 1149-1157 and 1636-1644), the topoisomerase-II-binding sites (1252-1266, 1456-1470) and an Alu element (site 2687-2998), were sequentially synthesized, forming an artificial splicing of the MAR fragment 369 bp long [Takara Biotechnology (Dailan) Co., Ltd, China]. The KpnI/BglII enzyme sites (GTCAGACTC and AGCGGTACC) were inserted at the two ends of the fragment.

Vector construction. The $2.15 \mathrm{~kb}$ MAR and $369 \mathrm{bp}$ MAR splice were inserted into the pMD18-T-vector (Takara Bio, Inc., Shiga, Japan). Following sequence identification, two MAR fragments and a pCATG plasmid were cut with KpnI and $B g l \mathrm{II}$, and then ligated using the T4 DNA ligase, to generate the pCATGM1and pCATGM2 vectors containing the $2.15 \mathrm{~kb}$ MAR and 369 bp MAR splice, respectively. The constructs are shown schematically in Fig. 1.

Cell culture and transfection. Chinese hamster ovary cells (CHO; Institute of Laboratory Animal Sciences, China) were inoculated in six-well plates at $2 \times 10^{6}$ cells/well. After $24 \mathrm{~h}$, the cells were transfected with pCATG, pCATGM1 and pCATGM2 vectors according to the manufacturer's instructions (Xiamen Sunma Biotechnology Co., Ltd. Xiamen, China). Following transfection for $48 \mathrm{~h}$, the cells were screened in DMEM supplemented with $800 \mathrm{mg} / \mathrm{ml} \mathrm{G} 418$ (Calbiochem, La Jolla, CA, USA) for 10 days. The cells were then screened with a maintenance concentration of $400 \mathrm{mg} / \mathrm{ml} \mathrm{G} 418$ for 10 days. When the colonies of stably transfected cells had formed, these were digested with $0.25 \%$ trypsin. Each group of cells was diluted and single colonies were selected. The colonies were then replanted in a culture bottle to continue the culturing process. The cells were collected when their density reached $80-90 \%$.

CAT expression assays. Cells were collected, adjusted to the same concentration of $1 \times 10^{6}$ cells $/ \mathrm{ml}$ by cell counting, and then washed three times with pre-cooled PBS. Lysis buffer ( $1 \mathrm{M}$; 5-fold diluted) was then added to the tubes. The tubes were gently agitated, left to stand at room temperature for $30 \mathrm{~min}$, and then centrifuged at $13,000 \mathrm{x} \mathrm{g}$ for $10 \mathrm{~min}$ at $4^{\circ} \mathrm{C}$. Approximately $200 \mu 1$ supernatant was extracted and placed into EP tubes. Negative and positive controls were set. CAT content was assayed using a CAT ELISA kit (Roche Applied Science, Indianapolis, IN, USA) according to the manufacturer's instructions.

Analysis of gene copy number. Real-time fluorescent quantitative PCR was used to analyze the gene copy number using

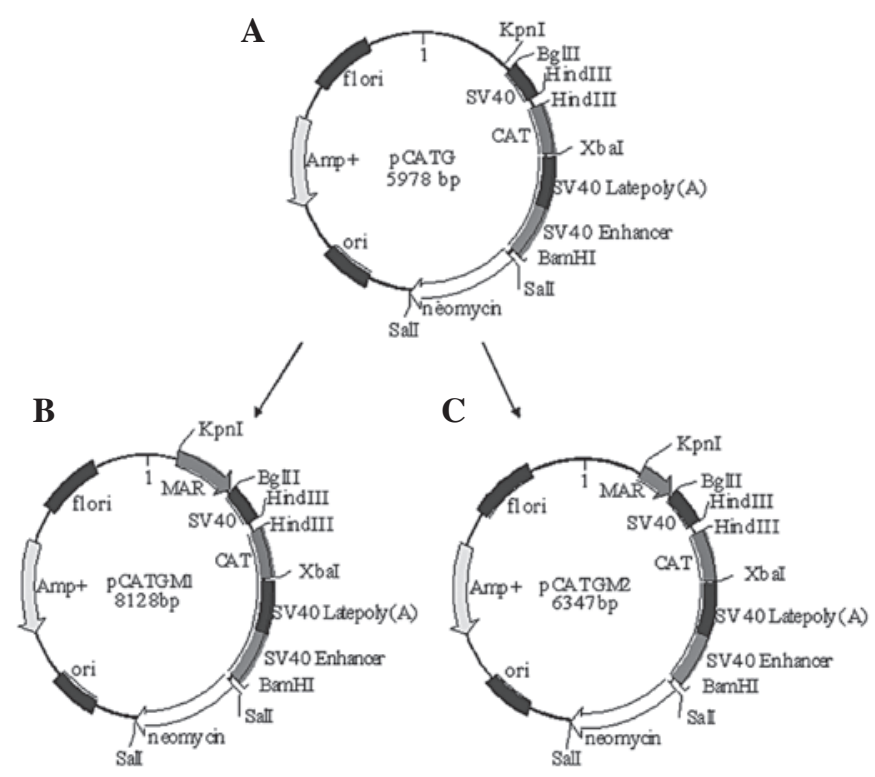

Figure 1. Plasmid vectors used in this study. Vectors are: (A) pCATG; (B) pCATGM1 and (C) pCATGM2.

internal control primers as the controls. Stably transfected cells were collected to extract the genomic DNA and served as PCR templates. The primers were designed according to the CAT gene sequence. The primers sequences used were: P1: 5'-CATCGCTCTGGAGTGAATACC-3' and P2: 5'-GGCATC AGCACCTTGTCG-3'. The internal control $\beta$-actin primer sequences were: P3: 5'-GTCTTTCTTCTGCCGTTCTC-3' and P4: 5'-ACCAGCCTCATTAGGTTTGT-3'. The PCR amplification system was $20 \mu 1$, and the PCR conditions were as follows: $95^{\circ} \mathrm{C}$ pre-degeneration for $10 \mathrm{~min}, 95^{\circ} \mathrm{C}$ for $15 \mathrm{sec}$ for 40 cycles, and $60^{\circ} \mathrm{C}$ for $1 \mathrm{~min}$. The SYBR-Green I fluorescent dye method was used for fluorescent quantitative real-time PCR. Experimental data were treated with $2^{-\Delta \Delta \mathrm{Ct}}$ (double $\Delta \mathrm{Ct}$ ) method. Each experiment was conducted in triplicate, and the mean $\mathrm{CT}$ and $\Delta \mathrm{Ct}$ values $[\Delta \mathrm{Ct}=\mathrm{Ct}$ (target gene) - $\mathrm{Ct}$ (internal gene)] were calculated. The $2^{-\Delta \Delta C t}$ was calculated, which is the relative fold of a gene copy number to the internal control gene copy number.

Statistical analysis. Analysis of variance was performed with SPSS 17.0 (SPPS, Inc., Chicago, IL, USA). The Q-test was used for comparison between two indices. $\mathrm{P}<0.05$ was considered to indicate statistical significance.

\section{Results}

Vector construction. The constructed vectors were digested with $K p n I$ and $K p n I / B g l I I$. The agarose gel electrophoresis results are shown in Fig. 1. Following digestion of the constructed pCATGM1 vector with $K p n \mathrm{I} / B g l \mathrm{II}$, an $\sim 2.15-\mathrm{kb}$ fragment appeared. Following digestion of the constructed pCATGM1 vector with KpnI, linear DNA was evident (Fig. 2). The results of the enzyme digestion conformed to the expected fragments. The results demonstrated that the vector containing MAR $(2.15 \mathrm{~kb})$ had been constructed. The same method was used to analyze the constructed pCATGM2 vector; a 369-bp 
Table I. CAT gene relative copy number analysis in stably transfected cell strains.

\begin{tabular}{lcccr}
\hline & Ct mean (vector) & Ct mean $(\beta$-actin) & $\Delta$ Ct mean & $\Delta \Delta$ Ct \\
\hline pCATG & 0.29277 & 20.94518 & 9.34759 & 0 \\
pCATGM1 & 32.24645 & 23.20043 & 9.04602 & 0.30157 \\
\hline
\end{tabular}

A

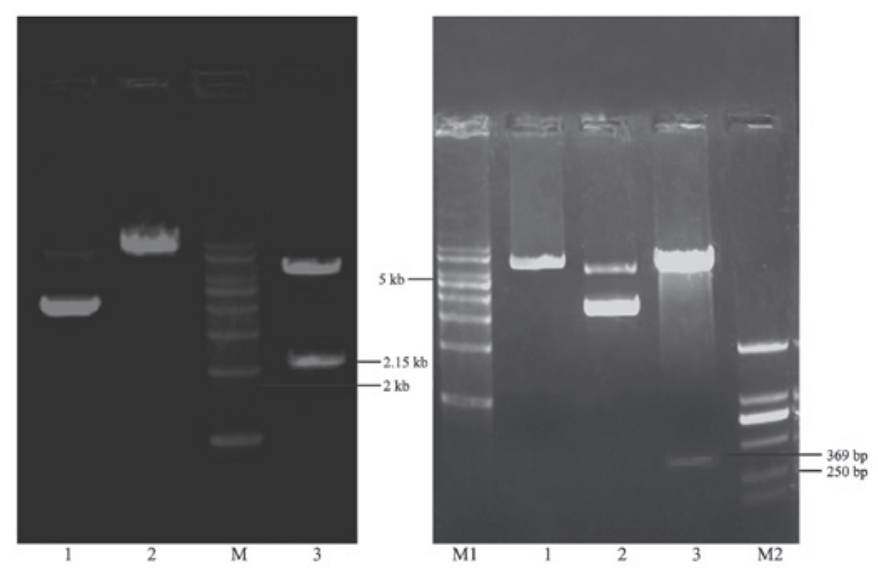

Figure 2. Identification of pCATGM1 and pCATGM2 using restriction enzymes. (A) pCATGM1 enzyme digestion. Lanes 1, no digestion; 2, KpnI digestion; 3, KpnI/BglIIdigestion; M, 1 kb DNA marker. (B) pCATGM2 enzyme digestion. Lanes 1, KpnI digestion; 2, no digestion; 3, KpnI/BglII digestion; M1, 1 kb DNA marker; M2, DL2000 DNA ladder.

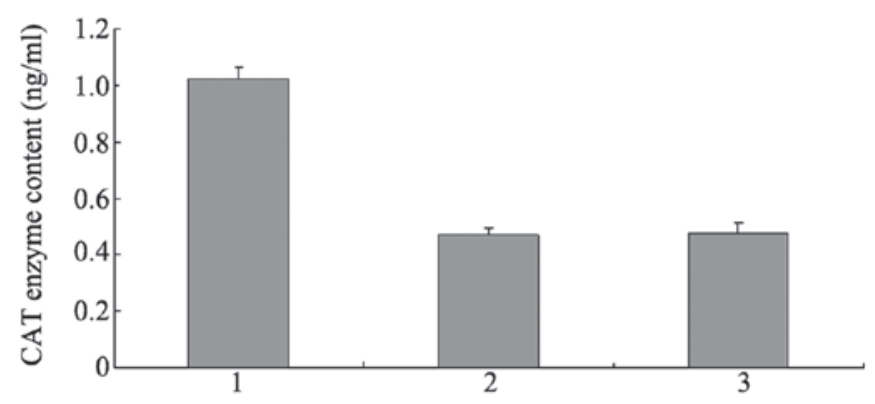

Figure 3. CAT content in different cells transfected with different vectors Lanes 1, pCATGM1 plasmid; 2, pCATGM2 plasmid; 3, pCATG plasmid.

long fragment and linear DNA were observed. The vector containing MAR (369 bp) had also been constructed.

CAT assays. CHO cells were transfected with the constructed vectors using the non-MAR pCATG plasmid as control. The cells were screened by the use of G418. When the cell concentration reached $80-90 \%$, the cells were adjusted to the same concentration of $1 \times 10^{6}$ cells $/ \mathrm{ml}$ via cell counting. The CAT content was analyzed following collection of the cells. The statistical analysis results from 10 monoclonal cells revealed that the CAT content of pCATGM1-transfected cells was 2.1489-fold higher than that of non-MAR pCATG-transfected cells. However, pCATGM2-transfected cells had the same CAT content as the non-MAR pCATG-transfected cells (Fig. 3). The difference in CAT expression between the different vector-transfected cells was statistically significant $(\mathrm{P}<0.01)$.
Gene copy number. Genomic DNA was extracted from six strains of cells from each group. Following real-time fluorescent quantitative PCR, $2^{-\Delta \Delta \mathrm{Ct}}$ was calculated. Combined with ELISA analysis, the data from Table I demonstrate that the CAT gene copy numbers of the pCATGM1 expression vector were 1.2-fold higher compared with those of the pCATG expression vector $(\mathrm{P}<0.01)$. In addition, the gene expression of the pCATGM1 expression vector was 2.1489-fold higher compared with that of the pCATG expression vector. Therefore, the CAT gene expression of the different strains of the pCATGM1 expression vector was not linear with its gene copy number. However, exogenetic gene expression enhancing may be correlated with the increase in gene copy number.

\section{Discussion}

Studies with regard to MAR increasing transgene expression have been reported $(6,15,16)$. Kim et al (9) inserted different MAR sources into the $\beta$-galactosidase gene expression vector and found that human $\beta$-globin MAR enhances the frequency of the positive colonies by 2.5 -fold, and the $\beta$-galactosidase gene expression by 7 -fold. However, other MAR sources have demonstrated little or no effect on $\beta$-galactosidase gene expression. Wang et al found that CAT gene expression was enhanced by 5.49 -fold when human $\beta$-globin MAR was inserted in the $5^{\prime}$ end of the CAT expression cassette (12). However, CAT gene expression decreased by 0.62 -fold when the $\beta$-globin MAR was inserted at the $3^{\prime}$ end of the CAT expression cassette. When the two ends of the CAT expression cassette were also inserted into the $\beta$-globin MARs, the CAT gene expression was 10.4-fold higher than that of the non-MAR expression vector (13). Wang et al found that if the two ends were inserted in the $\beta$-globin MAR and $\beta$-interferon MAR, the CAT gene expression was 4.5 -fold higher than that inserted only with $\beta$-globin MARs at the two ends of the CAT expression cassette, and 46.4-fold higher than that of the non-MAR expression vector (14). Due to the different sources of MAR, different effects of MAR were observed. Certain MAR sources have a strong effect on gene expression, whereas other sources have little effect. Another reason for the different effects may be the site of the MAR insertion. The $5^{\prime}$ end insertion possibly has more function than the $3^{\prime}$ end insertion.

In this study, we inserted longer $\beta$-globin MAR, containing connection DNA and all the characteristic elements in the $5^{\prime}$ end of the CAT expression cassette, thereby constructing the pCATGM1 expression vector. Following the transfection of $\mathrm{CHO}$ cells, we found that the pCATGM1 vector was capable of increasing CAT gene expression; the expression was 2.15-fold higher than that of the non-MAR expression vector. Therefore, human $\beta$-globin MAR is capable of enhancing the transgene 
expression and decreasing transgene silencing. Our results are consistent with those of Kim et al (9). The pCATGM2 vector, which only contained characteristic elements, was not able to increase the CAT gene expression, similar to that of the non-MAR expression vector. These results confirmed that although the characteristic elements of MAR may have an important effect on gene expression enhancing in theory, the characteristic elements of MAR alone do not have the same effect as that of the MAR sequence. Thus, more studies are required to identify which characteristic elements of MAR are involved in gene expression enhancing and which have no effect, and to determine whether the connection DNA between MARs is necessary for gene expression enhancement. Although the characteristic elements of MAR may have an important effect on gene expression, the connection DNA between the characteristic elements is necessary for the shape-formation and function of MAR. If the connection DNA between the characteristic elements is missing, MAR is not able to form the correct shape, and thus is not capable of enhancing gene expression.

Real-time fluorescent quantitative PCR was used to analyze the gene copy number. The results revealed that the pCATGM1 vector was capable of increasing the gene copy number by 1.2 -fold. In addition, $\beta$-globin MAR was able to enhance the transgene copy number. However, the CAT gene expression of different strains of the pCATGM1 expression vector was not linear with the gene copy number of the pCATGM1 expression vector. Thus, we deduced that exogenetic gene expression enhancement may be correlated with the increase in gene copy number. This result is not consistent with those of other studies $(11,12)$. Therefore, the mechanism requires further investigation.

An increasing number of separated MAR fragments has led to an improvement in our understanding of the function and mechanism of MAR. However, investigations concerning MAR are in their infancy. Although several studies have shown that MAR is able to increase gene expression, decrease transgene silencing and decrease the expression difference in different transformants, knowledge of MAR expression regulation at the chromatin level and the mechanism of its effect on transgene expression remains limited and should be investigated.

\section{References}

1. Spiker S and Thompson WF: Nuclear matrix attachment region and transgene expression in plants. Plant Physiol 110: 15-21, 1996.

2. Jenuwein T, Forrester WC, Fernández-Herrero LA, Laible G, Dull M and Grosschedl R: Extension of chromatin accessibility by nuclear matrix attachement regions. Nature 385: 269-272, 1997.

3. Zehnpfennig D, Deissler H, Polack A, Herr D, Bornkamm GW and Kurzeder C: B-cell-specific elements enhance sustained gene expression mediated by self-replicating extrachromosomal vectors. Mol Med Rep 3: 689-692, 2010.

4. Argyros O, Wong SP, Fedonidis C, et al: Development of S/MAR minicircles for enhanced and persistent transgene expression in the mouse liver. J Mol Med (Berl) 89: 515-529, 2011.

5. Girod PA, Nguyen DQ, Calabrese D, et al: Genome-wide prediction of matrix attachment regions that increase gene expression in mammalian cells. Nat Methods 4: 747-753, 2007.

6. Sjeklocha L, Chen Y, Daly MC, Steer CJ and Kren BT: $\beta$-globin matrix attachment region improves stable genomic expression of the Sleeping Beauty transposon. J Cell Biochem 112: 2361-2375, 2011.

7. Zhang J, Lu L, Ji L, Yang G and Zheng C: Functional characterization of a tobacco matrix attachment region-mediated enhancement of transgene expression. Transgenic Res 18: 377-385, 2009.

8. van der Geest AHM, Hall GE, Spiker S and Hall TC: The $\beta$-phaseolin gene is flanked by matrix attachment regions. Plant J 6: 413-423, 1994.

9. Kim JM, Kim JS, Park DH, et al: Improved recombinant gene expression in $\mathrm{CHO}$ cells using matrix attachment regions. J Biotechnol 107: 95-105, 2004.

10. Girod PA, Zahn-Zabal M and Mermod N: Use of the chicken lysozyme 5 ' matrix attachment region to generate high producer CHO cell lines. Biotechnol Bioeng 91: 1-11, 2005.

11. Buceta M, Galbete JL, Kostic C, Arsenijevic Y and Mermod N: Use of human MAR elements to improve retroviral vector production. Gene Ther 18: 7-13, 2011.

12. Wang TY, Yang R, Qin C, Wang L and Yang XJ: Enhanced expression of transgene in $\mathrm{CHO}$ cells using matrix attachment region. Cell Biol Int 32: 1279-1283, 2008.

13. Wang TY, Zhang JH, Jing CQ, Yang XJ and Lin JT: Positional effects of the matrix attachment region on transgene expression in stably transfected CHO cells. Cell Biol Int 34: 141-145, 2010.

14. Wang F, Wang TY, Tang YY, Zhang JH and Yang XJ: Different matrix attachment regions flanking a transgene effectively enhance gene expression in stably transfected Chinese hamster ovary cells. Gene 500: 59-62, 2012.

15. Harraghy N, Regamey A, Girod PA and Mermod N: Identification of a potent MAR element from the mouse genome and assessment of its activity in stable and transient transfections. J Biotechnol 154: 11-20, 2011.

16. Van der Geest AH, Welter ME, Woosley AT, et al: A short synthetic MAR positively affects transgene expression in rice and Arabidopsis. Plant Biotechnol J 2: 13-26, 2004. 